\title{
Gabor Kernels for Textured Image Representation and Classification
}

\author{
Hugo Hidalgo-Silva \\ CICESE-Ciencias de la Computación, \\ Km. 107 Carr. Tijuana-Eda, \\ Ensenada 22800 México \\ hugo@cicese.mx
}

\begin{abstract}
A Gabor based representation for textured images is proposed. Instead of the ordinary filter bank, a reproducing kernel representation is constructed consisting of a sum of several local reproducing kernels. The image representation coefficients are computed by a basis pursuit procedure, and are then considered as the feature vectors. The feature vectors are used to construct a kernel for a support vector classifier. Results are presented for a set of oriented texture images.
\end{abstract}

\section{Introduction}

Classification and segmentation of digital images based on the texture content is a fundamental problem in computer vision. A lot of different techniques have been considered in literature, from signal processing [1 $2 / 3$, to statistical modeling based 4 15. In the signal processing approach, the Gabor filter is the preferred building block. The textured image is submitted to a linear transform system named filter bank, consisting of a set of Gabor filters. The output of each filter is another image, applied then to a feature extractor. Several feature extractors are considered in literature [6, some use only the magnitude response, others the real component and some authors apply a full-wave rectifier, or a sigmoidal function to each filter's output. For every image, a large set of features are usually considered, those features are then applied to a classifier to identify the corresponding texture in the image.

In this work a Gabor based representation for the image is proposed. Instead of a filter bank, the image is considered as an element of a reproducing kernel Hilbert space. A kernel is constructed consisting of a sum of several local reproducing kernels. The image representation is assembled by a basis pursuit procedure, and the coefficients of the representation are taken as the feature vectors. The feature vectors are then used to construct a kernel for a support vector classifier. Results are presented for a set of test images from the Brodatz album. 


\section{Signal Representation}

The Gabor transform is a fundamental tool for signal representation and decomposition. When a function $f$ in $L^{2}(\mathbb{R})$ is used to represent an analog signal with finite energy, its Fourier transform

$$
F(\omega)=\int_{-\infty}^{\infty} e^{-i \omega t} f(t) d t
$$

reveals the spectral information of the signal. But the Fourier transform is not very useful for extracting information of the spectrum from a local observation of the signal $f$. The Gabor approach consider a window for time localization. The optimal window for time localization is obtained using a Gaussian function:

$$
g_{\sigma}(t)=\frac{1}{2 \sqrt{\pi \sigma}} e^{-\frac{t^{2}}{4 \sigma}}
$$

where $\sigma>0$ is fixed. Optimality is characterized in terms of the uncertainty principle [7].

If $f(t) \in L^{2}(\mathbb{R})$, the Gabor transform $F_{\mu, \sigma}(\omega)$ is the Fourier transform of the product $f(t) g_{\sigma}(t-\mu)$ :

$$
F_{\mu, \sigma}(\omega)=\int_{-\infty}^{\infty} e^{-j \omega t} f(t) g_{\sigma}(t-\mu) d t .
$$

The Gabor transform is also represented as $F_{\mu, \sigma}(\omega)=\left(\mathcal{G}_{\mu}^{\sigma} f\right)(\omega)$, and can be interpreted as a dot product relation that measures the similarity of $f(t)$ to the Gabor elementary function $(\mathrm{GEF}) G_{\mu, \omega}^{\sigma}(t)=g_{\sigma}(t-\mu) e^{j \omega t}[7]$ :

$$
\left(\mathcal{G}_{\mu}^{\sigma} f\right)(\omega)=\left\langle f, G_{\mu, \omega}^{\sigma}\right\rangle .
$$

Gabor elementary functions, also named as Gabor atoms or windowed Fourier atoms, have great applicability to spectral analysis of textures. Some authors consider only the real part of the GEF to construct filter banks employed in the classification task [16]. Texture analysis with Gabor filters is usually performed by obtaining a bank of Gabor filters tuned at different $\mu, \omega$ parameters. Then, a non linearity is applied to every Gabor function in order to select the proper feature of every filter. In this work, we are interested in the construction of a reproducing kernel Hilbert space (RKHS) for images with a kernel derived of the Gabor elementary functions. RKHS have acquired great importance in the learning community because they form the basis of the Kernel learning methodology [8]. By definition, a Hilbert space $\mathcal{H}$ es called a reproducing kernel Hilbert space if the following conditions are satisfied [10]:

1. the elements of $\mathcal{H}$ are (complex or real valued) functions defined on any set $\mathcal{D}$;

2. for every $t \in \mathcal{D}$ there exists $K_{t}>0$ such that

$$
\|f(t)\| \leq K_{t}|f| \quad f \in \mathcal{H} .
$$


In an RKHS $\mathcal{H}$, for every $t \in \mathcal{D}$ there is a function $R(\cdot, t) \in \mathcal{H}$ such that

$$
f(t)=\langle f, R(\cdot, t)\rangle \quad f \in \mathcal{H}
$$

by the Riesz representation theorem and the evaluation functionals are determined by the function $R(s, t)$ on $\mathcal{D} \times \mathcal{D}$, called the kernel of the RKHS $\mathcal{H}$. The kernel $R(s, t)$ of an RKHS is a symmetric and positive definite function, i.e. $R(t, s)=\overline{R(s, t)}$ and for any finite set $\left\{s_{i} \in \mathcal{D} ; i=1,2, \ldots\right\}$ and complex numbers $\lambda_{i}(i=1,2, \ldots, n)$,

$$
\sum_{i, j=1}^{n} \lambda_{i} \overline{\lambda_{j}} R\left(s_{i}, s_{j}\right) \geq 0 .
$$

Besides, the RKHS $\mathcal{H}$ with kernel $R$ is generated by $\{R(\cdot, t) ; t \in \mathcal{D}\}$. Another approach to construct a RKHS consist in considering the Loéve Transform [10]. For $x \in \mathcal{H}_{1}$, a closed subspace of $\mathcal{H}$ generated by $\{h(t) ; t \in \mathcal{D}\}$, the function $\hat{x}(t):=\langle x, h(t)\rangle$ is called the Loéve transform of $x$. The Loéve Transform form an RKHS with kernel

$$
R(s, t)=\langle h(t), h(s)\rangle \quad s, t \in \mathcal{D} .
$$

For example, considering a finite interval $[-A, A]$ and the subspace of $L^{2}[-A, A]$ generated by $h(t)=e^{-i \omega t}$ for $-\infty<t<+\infty$. In this case the kernel is

$$
\langle h(t), h(s)\rangle=\frac{1}{2 A} \int_{-A}^{A} e^{i \omega t} e^{-i \omega s} d \omega=\frac{\sin A(t-s)}{A(t-s)},
$$

and the Loéve transform of $F \in L^{2}[-A,+A]$ is the Fourier transform

$$
f(t)=\frac{1}{2 A} \int_{-A}^{A} e^{i \omega t} F(\omega) d \omega
$$

Then, the finite Fourier transforms with fixed band limit $A$ form an RKHS, the Hilbert space $L_{A}$ of band-limited signals. Here, we are interested in an RKHS for band-limited signals but considering the Gabor representation. Consider the kernel obtained with the dot product of the GEF's:

$$
\begin{array}{r}
Q(s, t)=\left\langle G_{\mu, \omega}^{\sigma}(s), G_{\mu, \omega}^{\sigma}(t)\right\rangle= \\
\frac{1}{2 A} \int_{-A}^{A} e^{i \omega t} e^{-i \omega s} g_{\sigma}(t-\mu) g_{\sigma}(s-\mu) d \omega= \\
g_{\sigma}(t-\mu) g_{\sigma}(s-\mu) \frac{\sin A(s-t)}{A(s-t)}
\end{array}
$$

by this formulation, we can represent an element of the Hilbert space of bandlimited signals in terms of the kernel (5). 


\section{Image Representation}

In the usual Gabor expansion texture recognition approach, a bank of Gabor filters is applied to a portion of the image [2. The basic even-symmetric Gabor filter oriented at $0^{\circ}$ is the real part of $G$ with a variance parameter for each axis $\sigma_{x}, \sigma_{y}$ :

$$
h(k, l)=e^{-\frac{1}{4}\left(\frac{k^{2}}{\sigma_{x}}+\frac{l^{2}}{\sigma_{y}}\right)} \cos \left(2 \pi f_{0} k\right),
$$

where $f_{0}$ is the radial center frequency. Other orientations are obtained by rotating the reference coordinate system $(k, l)$ some angle $\theta$. We consider the image representation in terms of a kernel:

$$
f(t)=\sum_{i=0}^{i=N} \beta_{i} \tilde{Q}\left(s_{i}, t\right),
$$

where $\tilde{Q}=\sum_{j=1}^{M} R_{j}(s, t)$.

Each $R_{j}(s, t)$ with different parameters $\mu_{x}, \mu_{y}, \sigma_{x}, \sigma_{y}, \theta . N$ is the number of pixels, $M$ the number of kernels and the space coordinates are two-dimensional $t=\left(t_{x}, t_{y}\right), s=\left(s_{x}, s_{y}\right)$. For the kernel construction we have used the property that a sum of kernels is a kernel 9 . Observe that the number of coefficients is the same as the number of image pixels, contrary to the filter bank approach, where we have a much more large feature set.

The selection of the $\beta$ coefficients is considered by a basis pursuit (BP) procedure [11. In the BP approach, the basis dictionary is assumed overcomplete, and there may exist many representations as (6). In our implementation, the kernel is constructed by a sum of several local kernels, and we assume that the representation may not be unique. The $\mathrm{BP}$ technique consist on finding a representation whose coefficients have a minimal $l^{1}$ norm. For that purpose, the minimization problem is:

$$
\min \|\beta\|_{1} \quad \text { subject to } \quad \boldsymbol{\Phi} \beta=\mathbf{d}
$$

with $\boldsymbol{\Phi}$ the Gram matrix of kernel evaluations $\boldsymbol{\Phi}_{i, j}=\tilde{Q}\left(s_{i}, t_{j}\right)$, $\mathbf{d}$ is the image representation in a lexicographically ordered vector. The minimization problem is implemented by solving the equivalent linear program

$$
\min \mathbf{c}^{t} \mathbf{x} \text { subject to } \mathbf{A x}=\mathbf{b}, \quad \mathbf{x} \geq 0 \text {, }
$$

making the translations $\mathbf{A}=(\boldsymbol{\Phi},-\boldsymbol{\Phi}), \mathbf{b}=\mathbf{d}, \mathbf{x}=(\mathbf{u}, \mathbf{v}), \beta=(\mathbf{u}-\mathbf{v})$, and considering $\mathbf{c}$ a vector of ones. The solution of (7) is obtained by solving the linear program (8). The linear program can be efficiently solved with public available software like PCx [12]. 


\section{Image Classification}

For the image classification process, the kernel representation (6) is obtained for every element of a set of sub-images extracted from the training and testing images. The corresponding coefficients of the kernel representation are employed to construct a kernel machine; a support vector classifier (SVC). The SVC is constructed assuming a kernel function $k(x, z)$ implementing a dot product among elements of a feature space: $\langle\Phi(x), \Phi(z)\rangle$. The usual kernel construction for image classification has been to perform dot products of sub-elements of the image, then raise the resulting dot product to a power $d_{1}$ to form a local kernel. The final kernel is the sum over the whole image of the local kernels [8]. In this work, the kernel is constructed as a function of the dot product of the image coefficients, $\left\langle\beta_{i}, \beta_{j}\right\rangle$. The SVC is aimed to construct the classifier function $c(x)=\operatorname{sign}\left(\sum_{i}^{l} y_{i} \alpha_{i} k\left(x, x_{i}\right)+b\right)$ in terms of a Gram matrix

$$
\mathbf{K}_{i, j}=k\left(x_{i}, x_{j}\right),
$$

by solving the system:

$$
\begin{array}{r}
\max W(\alpha)=\sum_{i=1}^{l} \alpha_{i}-\frac{1}{2} \sum_{i, j=1}^{l} \alpha_{i} \alpha_{j} y_{i} y_{j} k\left(x_{i}, x_{j}\right), \\
\text { subject to } \quad 0 \leq \alpha_{i} \leq \frac{C}{l} \quad \text { for all } \quad i=1, \ldots, l . \\
\text { and } \quad \sum_{i=1}^{l} \alpha_{i} y_{i}=0 .
\end{array}
$$

Where $l$ is the number of patterns, $y$ the corresponding output $( \pm 1)$ and $b$ is the threshold 8 . We construct our SVC kernel matrix $\mathbf{K}$ by performing the dot product among coefficients. Several other kernels can also be obtained using kernel properties [8]9].

\section{Application Results}

A set of 560 non-overlapping images of $40 \times 40$ pixels were extracted of the images observed in Fig. 1. The images correspond to textures D1,D11,D17,D20,D21,D22,

Table 1. Classification accuracy results for SVC's with different kernel functions

\begin{tabular}{|c|c|}
\hline kernel & Classification Accuracy (\%) \\
\hline$<x, y>$ & 41.7 \\
$\langle x, y\rangle^{2}$ & 67.85 \\
$\left\langle\beta_{i}, \beta_{j}\right\rangle$ & 36.26 \\
$\left.<\beta_{i}, \beta_{j}\right\rangle^{2}$ & 94.78 \\
$\left.<\beta_{i}, \beta_{j}\right\rangle^{3}$ & 52.47 \\
$\mathrm{RBF}$ & 86.26 \\
\hline
\end{tabular}




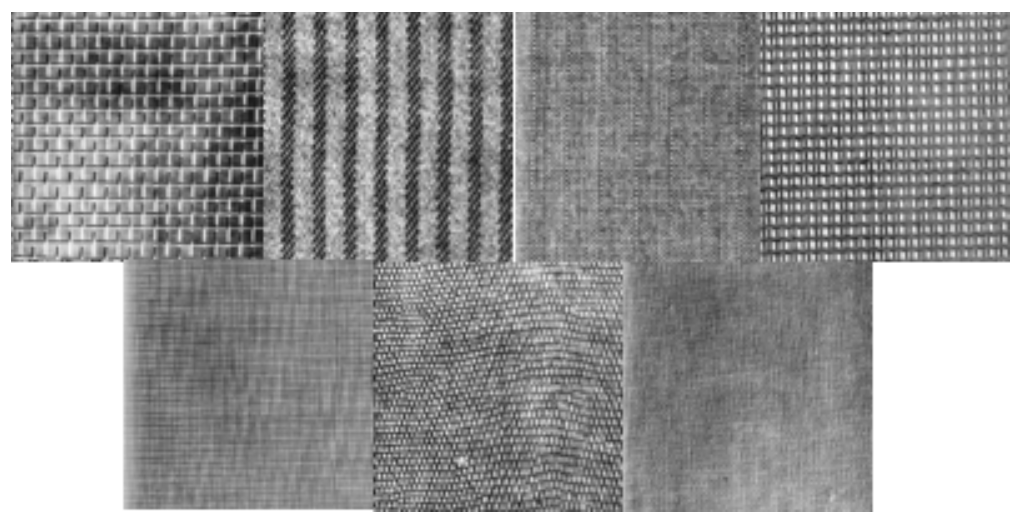

Fig. 1. Brodatz images (upper left to lower right) D1,D11,D17,D20,D21,D22 and D77

Table 2. Parameters of the kernel functions $R_{j}(s, t)$

\begin{tabular}{|c|c|c|c|c|c|c|c|c|c|c|c|}
\hline kernel & $\mu_{x}$ & $\mu_{y}$ & $\sigma_{x}$ & $\sigma_{y}$ & $\theta$ & kernel & $\mu_{x}$ & $\mu_{y}$ & $\sigma_{x}$ & $\sigma_{y}$ & $\theta$ \\
\hline 1 & 20 & 20 & 40 & 40 & 0 & 21 & 20 & 20 & 20 & 20 & 0 \\
2 & 20 & 20 & 40 & 10 & 45 & 22 & 20 & 20 & 20 & 5 & 45 \\
3 & 20 & 20 & 40 & 10 & 90 & 23 & 20 & 20 & 20 & 5 & 90 \\
4 & 20 & 20 & 40 & 10 & 135 & 24 & 20 & 20 & 20 & 5 & 135 \\
5 & 10 & 10 & 20 & 20 & 0 & 25 & 20 & 30 & 20 & 20 & 0 \\
6 & 10 & 10 & 20 & 5 & 45 & 26 & 20 & 30 & 20 & 5 & 45 \\
7 & 10 & 10 & 20 & 5 & 90 & 27 & 20 & 30 & 20 & 5 & 90 \\
8 & 10 & 10 & 20 & 5 & 135 & 28 & 20 & 30 & 20 & 5 & 135 \\
9 & 10 & 20 & 20 & 20 & 0 & 29 & 30 & 10 & 20 & 20 & 0 \\
10 & 10 & 20 & 20 & 5 & 45 & 30 & 30 & 10 & 20 & 5 & 45 \\
11 & 10 & 20 & 20 & 5 & 90 & 31 & 30 & 10 & 20 & 5 & 90 \\
12 & 10 & 20 & 20 & 5 & 135 & 32 & 30 & 10 & 20 & 5 & 135 \\
13 & 10 & 30 & 20 & 20 & 0 & 33 & 30 & 20 & 20 & 20 & 0 \\
14 & 10 & 30 & 20 & 5 & 45 & 34 & 30 & 20 & 20 & 5 & 45 \\
15 & 10 & 30 & 20 & 5 & 90 & 35 & 30 & 20 & 20 & 5 & 90 \\
16 & 10 & 30 & 20 & 5 & 135 & 36 & 30 & 20 & 20 & 5 & 135 \\
17 & 20 & 10 & 20 & 20 & 0 & 37 & 30 & 30 & 20 & 20 & 0 \\
18 & 20 & 10 & 20 & 5 & 45 & 38 & 30 & 30 & 20 & 5 & 45 \\
19 & 20 & 10 & 20 & 5 & 90 & 39 & 30 & 30 & 20 & 5 & 90 \\
20 & 20 & 10 & 20 & 5 & 135 & 40 & 30 & 30 & 20 & 5 & 135 \\
\hline
\end{tabular}

and D77 of Brodatz's album 13. For each class, 28 images were used as training, and 52 as testing data. The coefficients of (6) were obtained considering the kernel parameters shown on Table 1. Results for different kernel functions are presented in Table 2, observe that the first two kernels are constructed with dot products of the raw images.

From Table 2 observe also that using the dot products of the images or the dot product of coefficients alone produce similarly wrong results. The main 
improvement is obtained when the second order polynomial kernel is constructed. For the second order of the bare images an improvement to $67.85 \%$ is obtained. But when the second order polynomial kernel for the coefficients is constructed, an increase of accuracy to $94.78 \%$ is observed. The result for the radial basis function (RBF) kernel $k_{i, j}=e^{\left\|\beta_{i}-\beta_{j}\right\|^{2}}$ was obtained with parameter $\gamma=0.004$. All the results are presented for a $\nu \mathrm{SVC}$ with parameter $\nu=.5$.

\section{Conclusions}

An image representation is proposed to detect texture. The representation is based on an RKHS constructed using the Gabor elementary functions. The representation is assembled using a basis pursuit approach, and the coefficients are employed to implement kernels of support vector classifiers. Results are presented for a set extracted of Brodatz texture images, achieving $94 \%$ of classification accuracy.

\section{References}

1. Randen, T., Håkon Husøy, J.: Filtering for Texture Classification: A Comparative Study. IEEE Tran. on Pattern Analysis and Machine Intelligence, 21 (1989) 291-310.

2. Jain, A. K., Farrokhnia, F.: Unsupervised texture segmentation using Gabor Filters, Pattern Recognition,24 (1990) 1167-1186.

3. Teuner, A., Pichler O., Hosticka, B.J.: Unsupervised texture segmentation of images using tuned matched Gabor filters, IEEE Trans. Image Process. 4 (1995) 863-870.

4. Haralick, R.: Statistical and Structural Approaches to Texture, Proceedings of the IEEE, 67 (1979) 786-804

5. Varma, M., Zisserman, A.: A statistical Approach to Texture Classification from Single Images, International Journal of Computer Vision, 62 (2005) 61-81.

6. Clausi, D., Jernigan, M.: Designing Gabor filters for optimal texture separability. Pattern Recognition, 33 (2000) 1835-1849.

7. Chui, C.K.: An Introduction to Wavelets, San Diego, USA, Academic Press, (1992).

8. Schölkopf, B.,Smola, A.: Learning with Kernels, Cambridge, USA, MIT Press, (2002).

9. Shawe-Taylor, J., Cristianini, N. : Kernel Methods for Pattern Analysis, Cambridge, UK, Cambridge University Press, (2004).

10. Máté, L.: Hilbert Space Methods in Science and Engineering. Bristol, England: Adam Hilger (1989).

11. Chen, S.S., Donoho, D.L., Saunders, M.A.: Atomic Decomposition by Basis Pursuit, SIAM Review, 43 (2001) 129-159.

12. Czyzyk, J., Mehrotra, S., Wagner, M., Wright, S.: PCx User Guide, Optimization Technology Center, Technical Report OTC 96/01 (1997).

13. Brodatz, P. : Textures: A Photographic Album for Artists and Designers, Dover Publications, New York (1966). 